\title{
Life-threatening events in patients with pheochromocytoma
}

\author{
Anna Riester, Dirk Weismann', Marcus Quinkler², Urs D Lichtenauer³, \\ Sandra Sommerey ${ }^{4}$, Roland Halbritter ${ }^{5}$, Randolph Penning $^{6}$, Christine Spitzweg $^{7}$, \\ Jochen Schopohl, Felix Beuschlein and Martin Reincke \\ Medizinische Klinik und Poliklinik IV, Klinikum der Universität München, Ludwig-Maximilians-Universität, \\ Ziemssenstr. 1, D-80336 Munich, Germany, ${ }^{1}$ Medizinische Klinik und Poliklinik I, Universitätsklinikum Würzburg, \\ Würzburg, Germany, ${ }^{2}$ Endokrinologie in Charlottenburg, Berlin, Germany, ${ }^{3}$ Helios Klinik Schwerin, Schwerin, \\ Germany, ${ }^{4}$ Chirurgische Klinik und Poliklinik - Innenstadt, Klinikum der Universität München, Ludwig-Maximilians- \\ Universität München, Munich, Germany, ${ }^{5}$ Facharztpraxis, Pfaffenhofen, Germany, ${ }^{6}$ Institut für Rechtsmedizin and \\ ${ }^{7}$ Medizinische Klinik und Poliklinik II, Klinikum der Universität München, Ludwig-Maximilians-Universität, Munich, \\ Germany
}

\author{
Correspondence \\ should be addressed \\ to M Reincke \\ Email \\ martin.reincke@ \\ med.uni-muenchen.de
}

\section{Abstract}

Objective: Pheochromocytomas are rare chromaffin cell-derived tumors causing paroxysmal episodes of headache, palpitation, sweating and hypertension. Life-threatening complications have been described in case reports and small series. Systematic analyses are not available. We took an opportunity of a large series to make a survey.

Design and methods: We analyzed records of patients diagnosed with pheochromocytomas in three geographically spread German referral centers between 2003 and $2012(n=135)$.

Results: Eleven percent of the patients (ten women, five men) required in-hospital treatment on intensive care units (ICUs) due to complications caused by unsuspected pheochromocytomas. The main reasons for ICU admission were acute catecholamine induced Tako-Tsubo cardiomyopathy $(n=4)$, myocardial infarction $(n=2)$, acute pulmonary edema $(n=2)$, cerebrovascular stroke $(n=2)$, ischemic ileus $(n=1)$, acute renal failure $(n=2)$, and multi organ failure $(n=1)$. One patient required extracorporeal membrane oxygenation due to a hypertensive crisis with lung edema occurring during delivery $(n=1)$. Two patients died of refractory shock and pheochromocytomas were found postmortem. Two patients were treated by emergency surgery. Compared to pheochromocytoma patients without life-threatening events $(n=120)$, patients with complications had a significant larger maximal tumor diameter $(7.0 \mathrm{vs} 4.5 \mathrm{~cm}, P<0.01)$, higher levels of catecholamines (20- vs ninefold upper limit of normal, $P<0.01$ ), and tended to be younger (42 vs 51 years, $P=0.05$ ).

Conclusion: Although pheochromocytomas are rare, they are likely to be associated with a life-threatening situation.

Clinicians have to be aware of these situations and perform a timely diagnosis.

\section{Introduction}

Pheochromocytomas and paragangliomas are rare catecholamine-secreting tumors of chromaffin cell origin, which derive from the adrenal medulla or the extraadrenal sympathetic or parasympathetic nervous system. Clinically, pheochromocytomas present with symptoms associated with catecholamine excess, typically resulting in paroxysmal hypertension and the triad of headache, sweating, and palpitation. However, the presence and severity of these classical symptoms is highly variable $(1,2)$. In fact, up to $30 \%$ of the tumors are found incidentally (3) and autopsy studies indicate that many tumors are not identified during a lifetime $(4,5,6)$. The prevalence of pheochromocytomas in patients with hypertension is in the range of $0.6 \%$ (7). Pheochromocytomas can also present with life-threatening complications resulting in acute end organ damage $(8,9)$.

Published by Bioscientifica Ltd. 
Manipulation of the tumor during surgery or when intraabdominal pressure increases (for example, during pregnancy or vaginal delivery) can be a trigger that causes catecholamine release resulting in severe clinical consequences. However, the real incidence of life-threatening complications is unknown. Indeed, lack of any mortality is usually reported in the vast majority of series, and most life-threatening complications appear as anecdotal case reports in the literature. To our knowledge only two systematic studies have addressed this issue, thereby providing the basis of a better judgment of this situation $(10,11)$. In 2013, Scholten et al. (11) published a retrospective comprehensive study analyzing the perioperative management of pheochromocytoma crisis and recommended an elective adrenalectomy after cardiovascular stabilization and $\alpha$-blockade; 137 patients from one United States center were included and zero mortality was reported. In the same year, Giavarini et al. (10) presented a retrospective study including 140 patients with pheochromocytoma from a French center. They found catecholamine-induced cardiomyopathy in $11 \%$ of the patients and recommend screening for pheochromocytoma in patients with acute heart failure without evidence of valvular or coronary heart disease. Based on a review of 106 cases published between 2008 and 2013, Withelaw et al. (12) recently proposed a subclassification of pheochromocytoma crisis with 'type A crisis' characterized by a limited crisis with hemodynamic instability and end organ damage or dysfunction and 'type B crisis' defined as an extensive crisis with sustained hypotension (shock) and multi-organ dysfunction (two or more organ systems).

In the current study, we took the opportunity of three pheochromocytoma databases of referral hospitals in Munich, Würzburg, and Berlin. Pooled data analysis revealed a high incidence of complications. Using this data set we investigated clinical presentation and outcome of patients with life-threatening events causally in comparison to patients who underwent adrenalectomy for uncomplicated pheochromocytoma in the same time period.

\section{Subjects and methods}

We reviewed the records of patients with pheochromocytomas in three German tertiary referral centers: the University Hospital of the Ludwig-Maximilians-Universität, Munich $(n=72)$ and University Hospital Würzburg $(n=43)$ from January 2003 to December 2012 and the University Hospital of the Charite University, Berlin $(n=20)$, from January 2008 to December 2012. A shorter time period for the Charite University was chosen as only during this time a complete documentation of all cases of pheochromocytomas can be ensured. All 135 patients who underwent pheochromocytoma surgery during this period were included. Patients with repeated surgery due to relapse of malignant pheochromocytomas were included only for the first episode. We collected data of demographics, duration of hospitalization, symptoms, preoperative catecholamine levels, tumor histology, genetics, surgical treatment, and outcome. The patients were divided into two groups based on the presence or absence of life-threatening complications in their clinical course. A life-threatening complication was defined as a medical condition, requiring intensive care unit (ICU) admission most likely attributed to a catecholamine release of a hitherto undiagnosed pheochromocytoma or paraganglioma (pheo complication). According to this definition, in the three referral centers 15 out of 135 patients had a life-threatening event. Patients who underwent elective surgery due to uncomplicated pheochromocytomas $(n=120)$ served as a control group (pheo control).

Preoperative diagnosis was established by elevated plasma or urine catecholamines in combination with a positive tumor localization in computed tomography (CT), magnetic resonance imaging (MRI), metajodobenzylguanidin (MIBG) scintigraphy, or F-DOPA-PET-CT scan (positron emission tomography). For comparability, the highest level of catecholamines measured in an individual patient in urine or plasma was expressed as fold of upper limit of normal (ULN). The tumor size was determined either by pathology or by radiographic imaging, because tumor diameter in pathology was not provided consistently due to the retrospective design of the study. Mortality was defined as death during hospitalization. The clinical presentation of each patient prior to admission was assessed. Hypertension was defined either as treatment with antihypertensive medication or a previously documented elevated blood pressure above 140/90 mmHg. Paroxysmal hypertension, sweating, pallor, and palpitation were counted as pheochromocytoma specific symptoms. Malignancy was defined as the presence of distant metastases in organs other than sympathetic tissues (i.e., bone, liver, lung) (13).

\section{Ethical information}

Due to the retrospective character of the study, informed consent of the patients was not requested in accordance with German regulations. 
$|i||n| \ldots \ldots$<smiles>[AlH2]</smiles>
!n.

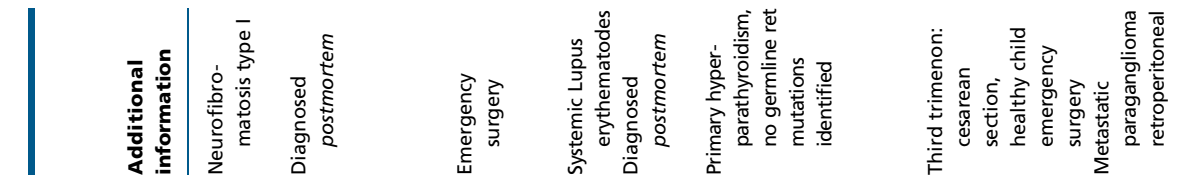
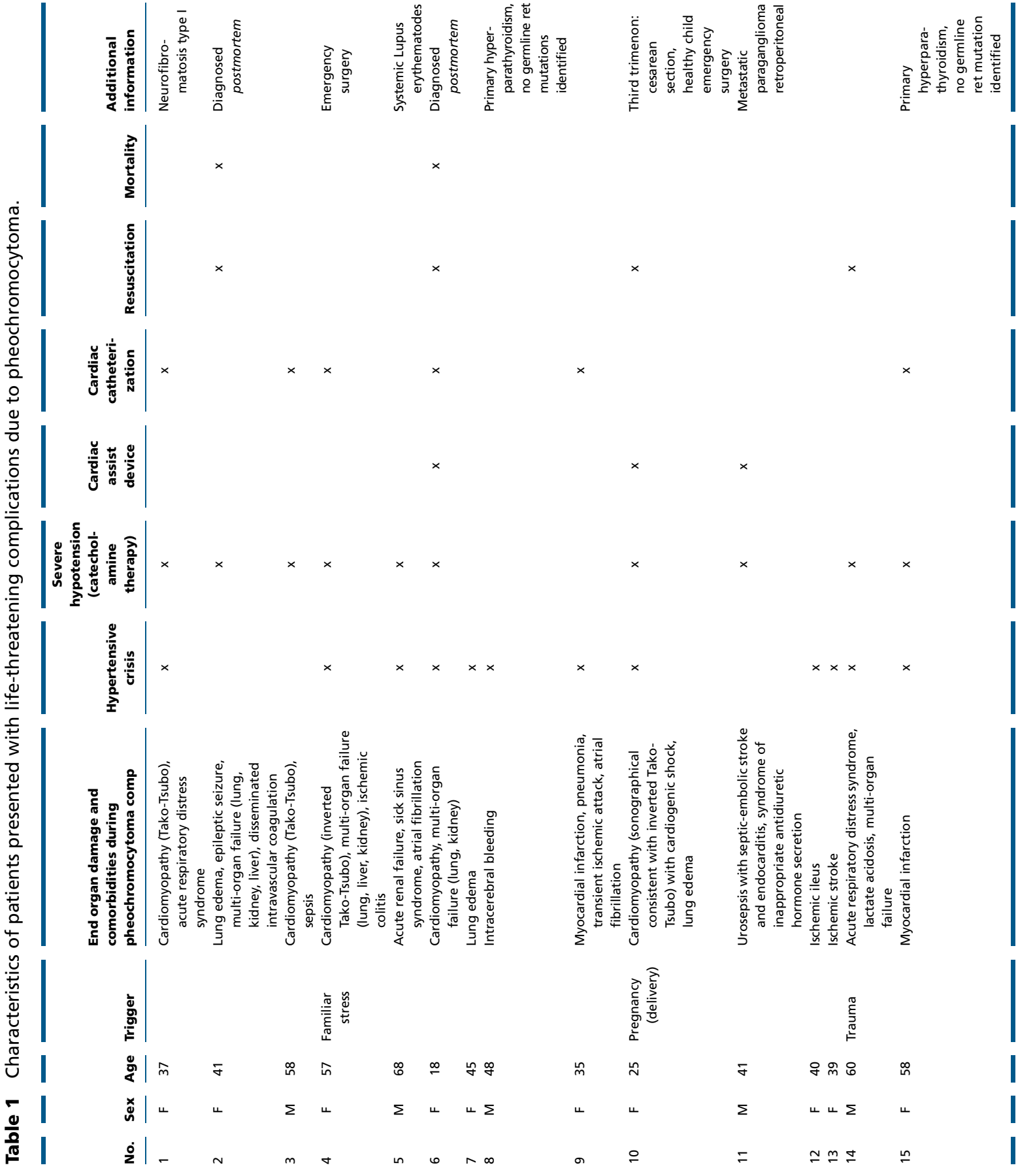

高

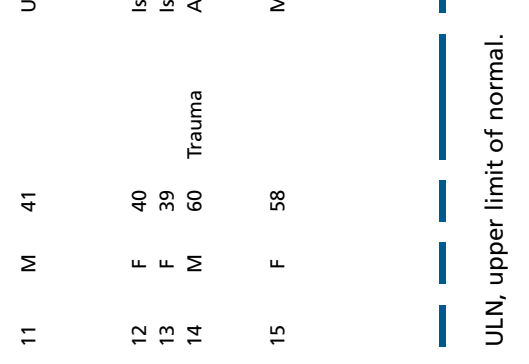




\section{Statistical analysis}

Comparison of binary variables was performed with Fisher's exact test after normality has been assessed. Continuous values are expressed as median (interquartile range) and were analyzed with the Mann-Whitney $U$ test. For multivariate analysis, factors significant in univariate analysis were inserted into a logistic regression analysis with backward-stepwise elimination. Statistical significance was determined using the SPSS Software Version 21 (SPSS, Inc., Chicago, IL, USA). Statistical significance was defined as $P<0.05$.

\section{Results}

Within the observation period, 135 pheochromocytoma patients underwent surgery in three German medical centers. Eleven percent (15 out of 135 patients) were treated in an intensive care setting due to a lifethreatening preoperative catecholamine crisis.

\section{Life-threatening events}

Six patients (40\%) had cardiovascular complications with suspected myocardial infarction leading to coronary angiography in all of the patients. Four patients had impaired left ventricular function with non-occluded coronaries leading to the diagnosis of catecholamine cardiomyopathy with Tako-Tsubo (-like) and inversed Tako-Tsubo features respectively (14). Two patients were diagnosed with myocardial infarction, and coronary stenosis was treated with percutaneous transluminal coronary angioplasty and stent implantation.

The other patients suffered from intestinal ischemia requiring resection of parts of the small intestine $(n=1)$, intracerebral hemorrhage $(n=1)$, embolic stroke $(n=1)$, acute renal failure $(n=1)$, urosepsis due to ureter compression by the tumor $(n=1)(15)$, life-threatening pulmonary edema and acute respiratory distress syndrome $(n=2)$, and multi-organ failure $(n=1)$ (Table 1$)$.

Furthermore, one patient had symptomatic uncontrollable high blood pressure during delivery after an uneventful pregnancy $(n=1)$. Preeclampsia was suspected and an emergency cesarean section was performed. A healthy boy was delivered, but the mother had to be resuscitated for more than $1 \mathrm{~h}$ and required extracorporeal membrane oxygenation (ECMO) due to acute heart and lung failure.

Using the above described classification by Whitelaw et al. (12), one-third of the patients suffered from a type A crisis (limited crisis) and two-thirds had a type B crisis (extensive crisis with shock).

\section{Mortality}

Two patients with type B crisis in the pheochromocytoma complication group (13\%) died of refractory shock. One patient was admitted to the hospital with cardiorespiratory shock requiring continuous life support. After stabilization for a few hours she died with unexplained multi-organ failure. Postmortem evaluation revealed a pheochromocytoma with a diameter of $8 \mathrm{~cm}$. The other patient presented with congestive heart failure and multiorgan failure after resuscitation due to repeated ventricular fibrillation. A percutaneous ventricular support device (Impella) was implanted; however, brain death had to be diagnosed 2 days after admission. Catecholamine excess was biochemically detected and an adrenal pheochromocytoma was confirmed by autopsy. No perioperative mortality was found in the pheo control group. In both groups no postoperative mortality (within 30 days after surgery) was detected.

\section{Triggers, clinical progress, and outcome of patients with pheochromocytoma-induced complications}

Circumstances likely to trigger these complications are sometimes identified. The following triggers might have induced life-threatening complications in our series: parturition $(n=1)$, trauma ( $n=1$; fall from a ladder), and emotional stress $(n=1)$ due to an unexpected death of the spouse.

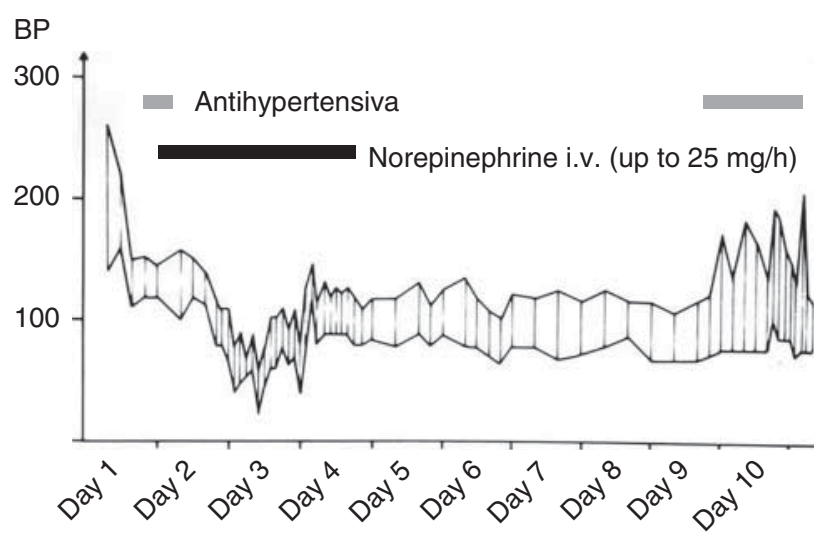

\section{Figure 1}

Blood pressure measurement in a patient with cardiomyopathy due to a pheochromocytoma. 
Twelve patients in the pheo complication group had at least one documented hypertensive crisis with a systolic blood pressure exceeding $180 \mathrm{mmHg}$. In contrast, during hospitalization two-thirds of the patients also required intravenous pressor application to treat severe shock. In four of the patients, hypertensive crisis was followed by severe hypotensive shock, which is exemplarily shown in Fig. 1. In three patients, the implantation of cardiac assist devices ( $n=1$, ECMO; $n=1$, intra-aortic balloon pump (IABP); $n=1$, Impella axial flow catheter) became necessary to improve cardiac output. Following cardiovascular stabilization, the patients received preoperative $\alpha$-blockade whenever possible.

Two patients did not achieve cardiorespiratory stabilization and underwent emergency adrenalectomy under an intensive care setting with continued mechanical respiration and/or continuous renal replacement therapy. One suffered from multi-organ failure including acute renal and liver failure, as well as ischemic colitis. The other was found to have Tako-Tsubo cardiomyopathy and acute respiratory distress syndrome.

\section{Comparison of patients with and without pheochromocytoma-induced complications}

Patients of the pheo complication group showed a trend toward younger age and had significantly higher levels of catecholamines and larger tumors (Table 2). Accordingly, maximal preoperative systolic and diastolic blood pressure was significantly higher in pheo complications compared to controls. The preoperative dose of phenoxybenzamine did not differ between the groups. To identify predictors of pheo complication, logistic regression analysis was performed. Only preoperative systolic blood pressure $(P<0.05$, OR 1.1) and the maximal tumor diameter $(P<0.05$, OR 2.3$)$ remained significant predictors of lifethreatening events.

The hospital stay was much longer in pheo complication (Fig. 2).

\section{Discussion}

Pooling results from three German tertiary hospitals resulted in the finding of a high incidence of life-

Table 2 Comparison of characteristics, clinical presentation, treatment, and outcome of the pheo controls with pheo complications, consisting of patients with life-threatening events leading to a preoperative treatment on the ICU. As characteristic symptoms, paroxysmal hypertension, headache, palpitations, and sweating was counted. Results are expressed as mean \pm s.E.M. for normally distributed variables or median (interquartile range) for non-normally distributed variables.

\begin{tabular}{l}
\hline \\
\hline$n$ \\
Sex (female) \\
Age (years) \\
Genetic syndrome (history) \\
Symptoms and diagnosis \\
Hypertension (history) \\
BP max systolic (mmHg) \\
BP max diastolic (mmHg) \\
Characteristic pheo symptoms \\
Catecholamines fold ULN \\
Primarily epinephrine producing \\
Tumor details \\
Extra-adrenal tumor \\
Tumor site (right) \\
Maximal tumor diameter (cm) \\
Malignancy \\
Necrosis at histology \\
Hemorrhage at histology \\
Treatment and outcome \\
Preoperative $\alpha$-blockade \\
Daily dose (phenoxybenzamine in mg) \\
Type of surgery (laparoscopic) \\
Postoperative ICU admission \\
Rehabilitation treatment \\
Mortality
\end{tabular}

\begin{tabular}{c}
\hline Pheo controls \\
\hline 120 \\
$48 \%$ \\
$51(22)$ \\
$20 \%$ \\
\\
$81 \%$ \\
$165(80)$ \\
$95(19)$ \\
$48 \%$ \\
$9(14)$ \\
$52 \%$ \\
$7 \%$ \\
$58 \%$ \\
$4.5(3)$ \\
$7 \%$ \\
$12 \%$ \\
$31 \%$ \\
$98 \%$ \\
$80(60)$ \\
$78 \%$ \\
$53 \%$ \\
$3 \%$ \\
$0 \%$ \\
\hline
\end{tabular}

\begin{tabular}{c}
\hline Pheo complications \\
\hline 15 \\
$67 \%$ \\
$42(21)$ \\
$13 \%$ \\
$67 \%$ \\
$235(35)$ \\
$120(10)$ \\
$13 \%$ \\
$20(31)$ \\
$71 \%$ \\
$7 \%$ \\
$50 \%$ \\
$7(4)$ \\
$7 \%$ \\
$14 \%$ \\
$14 \%$ \\
$100 \%{ }^{a}$ \\
$100(110)$ \\
$70 \%{ }^{a}$ \\
$100 \%^{a}$ \\
$60 \%{ }^{a}$ \\
$13 \%$ \\
\hline
\end{tabular}

\begin{tabular}{cc}
\hline $\boldsymbol{P}$ \\
\\
0.18 \\
0.05 \\
0.74 \\
\\
0.30 \\
$<0.01$ \\
$<0.001$ \\
$<0.05$ \\
$<0.01$ \\
0.16 \\
\\
1.00 \\
0.58 \\
$<0.01$ \\
1.00 \\
1.00 \\
0.66 \\
\\
1.00 \\
0.25 \\
0.70 \\
$<0.001$ \\
$<0.001$ \\
$<0.05$ \\
\end{tabular}

${ }^{a} n=13$, all patients of the pheo complication group who underwent adrenalectomy. 


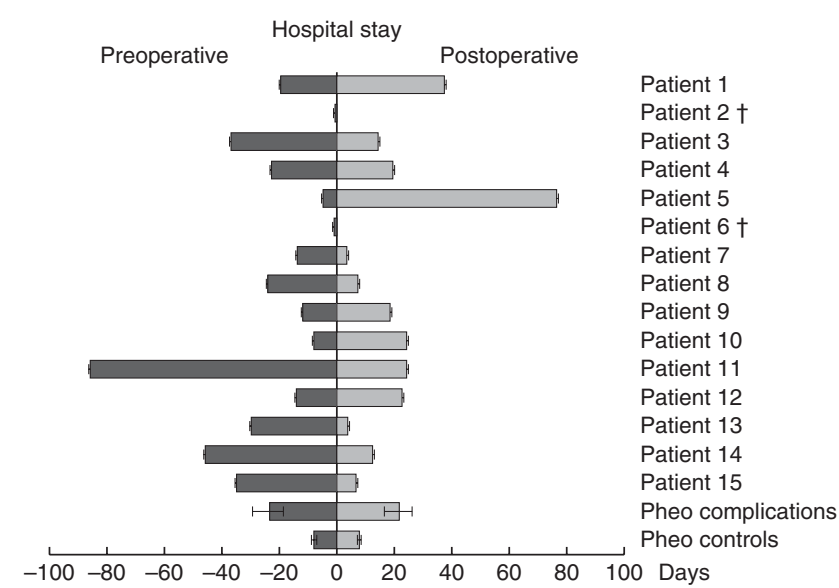

Figure 2

Duration of hospital stay of each patient of the pheo complications vs the median of hospital stay in the patients of the pheo controls. tdeceased patients.

threatening events due to pheochromocytoma and a higher mortality rate in this patient group than generally appreciated. Most life-threatening complications are described as anecdotal case reports and their incidence and outcome is variously reported.

Cardiovascular complications, such as hypertensive crisis and catecholamine-induced cardiomyopathy, are known to be the most frequent causes of life-threatening events in pheochromocytoma patients as summarized in a recently published review of the literature (12). Additionally, Giavarini et al. (10) reported that $11 \%$ of pheochromocytoma patients present with acute catecholamineinduced cardiomyopathy. In agreement with these studies, four of our patients suffered from pheochromocytoma-induced Tako-Tsubo cardiomyopathy and presented with the sequence of hypertensive crisis followed by hypotensive shock. A similar scenario is seen in other ICU patients following administration of very high doses of catecholamines. The pathomechanism for this clinical observation remains uncertain; however, there are several hypotheses to explain the hypotensive shock after exposure to high levels of catecholamines. Desensitization of adrenoreceptors, constricted circulatory volume, vasodilatation by abrupt withdrawal of norepinephrine and catecholamine-induced cardiotoxic effects leading to a left ventricular failure have been discussed as possible mechanisms $(8,16,17)$.

Catecholamine crisis during pregnancy or labor is certainly a unique clinical situation and especially demanding for treating physicians, because this scenario is associated with high morbidity and mortality for both mother and child $(18,19)$. To consider a pheochromocytoma in this situation is a particular diagnostic challenge, as preeclampsia is a much more common cause of hypertension in this patient group $(20,21)$.

The treatment goal is always to remove the source of catecholamine excess. Laparoscopic adrenalectomy following $\alpha$-blockade is considered the preferred surgical technique $(22,23)$. Scholten et al. (11) have recommended stabilization of the patients and elective surgery as an alternative therapeutic strategy. Although this can be considered a safe approach in some instances, delayed surgery seems not to be an option in all cases. In fact, two patients in our series had to undergo emergency adrenalectomy because of cardiorespiratory instability.

As described above, hemodynamic instability can be a severe problem in pheochromocytoma patients. Hypertension is recommended to be treated with $\alpha$-blockers and, if necessary, with other antihypertensive drugs as vasodilatators and calcium channel blockers (24). $\alpha$-blockers are associated with improved survival in the literature (12) although large prospective - or even randomized - series are missing. Treatment of hypotensive shock in patients with pheochromocytoma can be an even more demanding emergency situation. The recommended treatment is comprised of volume substitution and administration of very high doses of catecholamines. Additionally, vasopressin, which is not acting via adrenergic receptors, can be considered. A recent review of the literature indicates that circulatory support is strongly associated with the survival of extensive crisis with shock (survival 92\% vs $44 \%)$ (12). The circulatory support might enable the use of $\alpha$-blockers to fight the underlying pathological process. Nevertheless, fatal outcome is still possible as exemplified in one of our patients who died despite circulatory support. The pheochromocytoma complication rate reported in Scholten et al. (11) was 18\%. However, taking into account only those patients who required ICU treatment, the prevalence drops to $10 \%$, which is comparable to the $11 \%$ found in our study. This figure may underestimate the true frequency of pheochromocytoma-induced complications as autopsy studies indicate a higher prevalence than those reported within clinical studies. Nevertheless, the incidence of life-threatening complications in our pooled data analysis is surprisingly high, potentially caused by a selection bias of referral centers.

The mortality in our series was $13 \%$ (two of 15), which is in line with a reported overall mortality of $15 \%$ in a review of 106 cases (12). Interestingly, in both published 
series the perioperative mortality was $0 \%$. In the series published by Scholten et al. (11), only those patients were included if they had been referred to surgery. Applying similar criteria to our cohort would result in exclusion of two deceased patients and, thus, would also eliminate perioperative mortality in our series.

\section{Strengths and limitations of the study}

This study is a useful contribution to enlarge our knowledge on life-threatening situations in pheochromocytoma patients. The strengths of this study are the size of the cohort, inclusion of all other patients with pheochromocytomas treated during the study period in the centers as controls, and participation of three geographically spread German referral centers. Limitations include the potential underestimation of the true prevalence of life-threatening complications because of unrecognized lethal cases or a potential overestimation because of center-specific referral bias. Moreover, due to the retrospective character of the study we were not able to standardize the measurements of hormonal parameters.

\section{Perspective}

Taken together, in our retrospective study every tenth pheochromocytoma patient presented with a life-threatening complication. Although pheochromocytoma is a rare disease, it is likely to be associated with a lifethreatening situation. Clinicians should be aware of these situations and aim at a timely diagnosis. Specifically, clinicians should consider pheochromocytomas, especially in young patients with Tako-Tsubo cardiomyopathy, and in patients with a clinical course of hypertensive crisis followed by severe hypotensive shock.

\section{Declaration of interest}

The authors declare that there is no conflict of interest that could be perceived as prejudicing the impartiality of the research reported.

\section{Funding}

This research did not receive any specific grant from any funding agency in the public, commercial or not-for-profit sector.

\section{References}

1 Lenders JW, Eisenhofer G, Mannelli M \& Pacak K. Phaeochromocytoma. Lancet 2005366 665-675. (doi:10.1016/S0140-6736(05)67139-5)
2 Manger WM. An overview of pheochromocytoma: history, current concepts, vagaries, and diagnostic challenges. Annals of the New York Academy of Sciences 20061073 1-20. (doi:10.1196/annals. 1353.001)

3 Kopetschke R, Slisko M, Kilisli A, Tuschy U, Wallaschofski H, Fassnacht M, Ventz M, Beuschlein F, Reincke M, Reisch N et al. Frequent incidental discovery of phaeochromocytoma: data from a German cohort of 201 phaeochromocytoma. European Journal of Endocrinology/European Federation of Endocrine Societies 2009161 355-361. (doi:10.1530/EJE-09-0384)

4 Lo CY, Lam KY, Wat MS \& Lam KS. Adrenal pheochromocytoma remains a frequently overlooked diagnosis. American Journal of Surgery 2000179 212-215. (doi:10.1016/S0002-9610(00)00296-8)

5 Platts JK, Drew PJ \& Harvey JN. Death from phaeochromocytoma: lessons from a post-mortem survey. Journal of the Royal College of Physicians of London 199529 299-306.

6 Cohen DL, Fraker D \& Townsend RR. Lack of symptoms in patients with histologic evidence of pheochromocytoma: a diagnostic challenge. Annals of the New York Academy of Sciences 20061073 47-51. (doi:10.1196/annals.1353.005)

7 Omura M, Saito J, Yamaguchi K, Kakuta Y \& Nishikawa T. Prospective study on the prevalence of secondary hypertension among hypertensive patients visiting a general outpatient clinic in Japan. Hypertension Research 200427 193-202. (doi:10.1291/hypres.27. 193)

8 Prejbisz A, Lenders JW, Eisenhofer G \& Januszewicz A. Cardiovascular manifestations of phaeochromocytoma. Journal of Hypertension 201129 2049-2060. (doi:10.1097/HJH.0b013e32834a4ce9)

9 Brouwers FM, Eisenhofer G, Lenders JW \& Pacak K. Emergencies caused by pheochromocytoma, neuroblastoma, or ganglioneuroma. Endocrinology Metabolism Clinics of North America 200635 699-724, viii. (doi:10.1016/j.ecl.2006.09.014)

10 Giavarini A, Chedid A, Bobrie G, Plouin PF, Hagege A \& Amar L. Acute catecholamine cardiomyopathy in patients with phaeochromocytoma or functional paraganglioma. Heart 201399 1438-1444. (doi:10.1136/ heartjnl-2013-304073)

11 Scholten A, Cisco RM, Vriens MR, Cohen JK, Mitmaker EJ, Liu C, Tyrrell JB, Shen WT \& Duh QY. Pheochromocytoma crisis is not a surgical emergency. Journal of Clinical Endocrinology and Metabolism 201398 581-591. (doi:10.1210/jc.2012-3020)

12 Whitelaw BC, Prague JK, Mustafa OG, Schulte KM, Hopkins PA, Gilbert JA, McGregor AM \& Aylwin SJ. Phaeochromocytoma [corrected] crisis. Clinical Endocrinology 201480 13-22. (doi:10.1111/ cen.12324)

13 Lenders JW, Duh QY, Eisenhofer G, Gimenez-Roqueplo AP, Grebe SK, Murad MH, Naruse M, Pacak K, Young WF Jr \& Endocrine Society. Pheochromocytoma and paraganglioma: an endocrine society clinical practice guideline. Journal of Clinical Endocrinology and Metabolism 2014 99 1915-1942. (doi:10.1210/jc.2014-1498)

14 Kawai S, Kitabatake A \& Tomoike H. Guidelines for diagnosis of Takotsubo (ampulla) cardiomyopathy. Circulation Journal $2007 \mathbf{7 1}$ 990-992. (doi:10.1253/circj.71.990)

15 Tanriver Y, Betz MJ, Nibbe L, Pfluger T, Beuschlein F \& Strowski MZ. Sepsis and cardiomyopathy as rare clinical manifestations of pheochromocytoma - two case report studies. Experimental and Clinical Endocrinology \& Diabetes 2010118 747-753. (doi:10.1055/s-00301253413)

16 Costa VM, Carvalho F, Bastos ML, Carvalho RA, Carvalho M \& Remiao F. Contribution of catecholamine reactive intermediates and oxidative stress to the pathologic features of heart diseases. Current Medicinal Chemistry 201118 2272-2314. (doi:10.2174/ 092986711795656081)

17 Paur H, Wright PT, Sikkel MB, Tranter MH, Mansfield C, O'Gara P, Stuckey DJ, Nikolaev VO, Diakonov I, Pannell L et al. High levels of circulating epinephrine trigger apical cardiodepression in a $\beta 2$-adrenergic receptor/Gi-dependent manner: a new model of 
Takotsubo cardiomyopathy. Circulation 2012126 697-706. (doi:10.1161/CIRCULATIONAHA.112.111591)

18 Lenders JW. Pheochromocytoma and pregnancy: a deceptive connection. European Journal of Endocrinology/European Federation of Endocrine Societies 2012166 143-150. (doi:10.1530/EJE-11-0528)

19 Jozwik-Plebanek K, Peczkowska M, Klisiewicz A, Wrzesinski K, Prejbisz A, Niewada M, Kabat M, Szperl M, Eisenhofer G, Lenders JW et al. Pheochromocytoma presenting as takotsubo-like cardiomyopathy following delivery. Endocrine Practice 201420 e233-e236.

20 Plu I, Sec I, Barres D \& Lecomte D. Pregnancy, cesarean, and pheochromocytoma: a case report and literature review. Journal of Forensic Sciences 201358 1075-1079. (doi:10.1111/1556-4029.12107)

21 Biggar MA \& Lennard TW. Systematic review of phaeochromocytoma in pregnancy. British Journal of Surgery 2013100 182-190. (doi:10.1002/ bjs.8976)
22 Weismann D, Fassnacht M, Weinberger F, Hamelmann W, Diehl S, Lorenz K, Baerlehner E, Reincke M, Beuschlein F, Knoefel W et al. Intraoperative haemodynamic stability in patients with phaeochromocytoma - minimally invasive vs conventional open surgery. Clinical Endocrinology 200665 352-358. (doi:10.1111/ j.1365-2265.2006.02603.x)

23 Shen WT, Grogan R, Vriens M, Clark OH \& Duh QY. One hundred two patients with pheochromocytoma treated at a single institution since the introduction of laparoscopic adrenalectomy. Archives of Surgery 2010145 893-897. (doi:10.1001/archsurg.2010.159)

24 Mazza A, Armigliato M, Marzola MC, Schiavon L, Montemurro D, Vescovo G, Zuin M, Chondrogiannis S, Ravenni R, Opocher G et al. Anti-hypertensive treatment in pheochromocytoma and paraganglioma: current management and therapeutic features. Endocrine 2014 45 469-478. (doi:10.1007/s12020-013-0007-y)

Received 11 May 2015

Revised version received 10 August 2015

Accepted 7 September 2015 